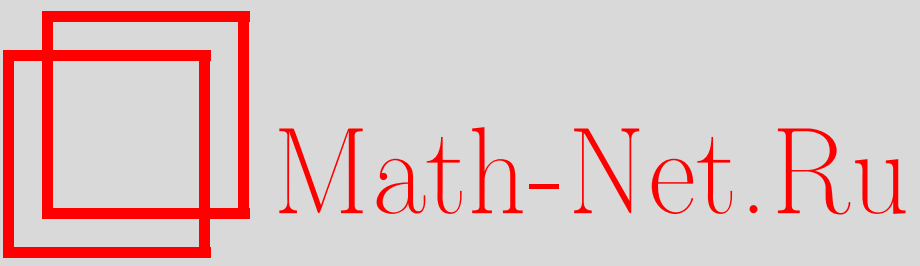

А. Б. Борисов, С. А. Зыков, М. В. Павлов, Уравнение Цицейки и размножение нелинейных интегрируемых уравнений, ТМФ, 2002, том 131, номер 1, 126-134

DOI: https://doi.org/10.4213/tmf319

Использование Общероссийского математического портала Math-Net.Ru подразумевает, что вы прочитали и согласны с пользовательским соглашением

http://www . mathnet.ru/rus/agreement

Параметры загрузки:

IP: 3.85 .73 .92

26 апреля 2023 г., 16:36:51

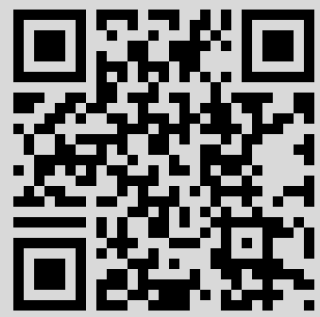


ТЕОРЕТИЧЕСКАЯ

И МАТЕМАТИЧЕСКАЯ

ФИЗИКА

Том 131, № 1

апрель, 2002

(C) 2002 г. $\quad$ А.Б. Борисов*, С. А. Зыков* ${ }^{*}$ М.В. Павлов ${ }^{\dagger}$

\section{УРАВНЕНИЕ ЦИЦЕЙКИ И РАЗМНОЖЕНИЕ НЕЛИНЕЙНЫХ ИНТЕГРИРУЕМЫХ УРАВНЕНИЙ}

Обсуждается метод одевания для уравнения Цицейки. Процедурой размножения получено модифицированное уравнение Цицейки.

\section{1. ВВЕДЕНИЕ}

В 1940 г. Шредингером при исследовании класса точно решаемых потенциалов в квантовой механике был предложен метод факторизации [1], [2]. Согласно этому методу собственные значения оператора $L=-\partial_{x}^{2}+u$ могут быть определены представлением его в виде $L_{0}=a_{0}^{+} a_{0}+\lambda_{0}$ с некоторым числом $\lambda_{0}$. При известном виде операторов $a_{0}$ определим оператор $L_{1}=a_{0} a_{0}^{+}+\lambda_{0}$ и представим его снова в виде $L_{1}=a_{1}^{+} a_{1}+\lambda_{1}=-\partial_{x}^{2}+u_{1}$, где $u_{1}=u-2 f_{x}$, и т.д. В теории солитонов спектральная задача, связанная с оператором Шредингера, ассоциирована с уравнением Кортевега-де Фриза, и последнее соотношение известно как преобразование Дарбу, а функция $f$ удовлетворяет модифицированному уравнению Кортевега-де Фриза. Обобщением метода факторизации является метод одеваюших цепочек, позволяюший не только получать потенциалы с бесконечным числом связанных состояний [3], которые не укладываются в рамки традиционных схем обратной задачи рассеяния, но и исследовать классы солитонных решений с нетривиальным асимптотическим поведением на бесконечности. В работе [4] была предложена процедура размножения интегрируемых уравнений, которая с помощью преобразований Дарбу и метода одевающих цепочек позволяет по известному интегрируемому уравнению и его $L-A$-паре получить не только новое интегрируемое уравнение, но и ассоциируемую с ним $L-A$-пару.

В этой работе мы применяем процедуру размножения [4] к уравнению Цицейки

$$
\theta_{x t}=\tau e^{2 \theta}-e^{-\theta}
$$

с произвольным параметром $\tau$ для вешественного поля $\theta(x, t)$. Многочисленные приложения этого уравнения в различных областях математики и физики были суммированы

* Институт физики металлов УрО РАН, Екатеринбург, Россия. E-mail: borisov@imp.uran.ru; Serg@imp.uran.ru

${ }^{\dagger}$ Московский государственный авиационный технологический университет, Москва, Россия 
в работе [5], и здесь мы упомянем некоторые из них. Уравнение (1) первоначально было получено в геометрии в 1907 г. Цицейкой при исследовании поверхностей, общая кривизна которых пропорциональна четвертой степени расстояния от фиксированной точки в касательной плоскости [6]. Оно появляется также в одномерной газовой динамике, когда уравнение Эйлера редуцируется к уравнению (1) при определенном уравнении состояния. Кроме того, уравнение Цицейки является редукцией двумерной решетки Тоды, уравнений Хироты-Сатцумы и нелинейной $S L(3, R)$-модели.

Уравнение (1) было переоткрыто в теории солитонов в конце семидесятых годов. Додд и Буллаф [7] исследовали уравнения типа $u_{x t}=f(u)$, которые допускают бесконечный набор интегралов движения. Они показали, что с точностью до точечных преобразований функция $f(u)$ может быть равной $u$ (уравнение Даламбера), ехр $u$ (Лиувилля), $\sin u$ (синус-Гордон) и $\exp (2 u)+\exp (-u)$ (Цицейки). Аналогичные и более полные результаты были независимо получены Жибером и Шабатом [8], которые в явном виде записали высшие симметрии этих уравнений. $L-A$-пара Лакса для уравнения Цицейки была найдена Михайловым [9].

В разделе 2 настоящей статьи, который частично носит методический характер, мы применяем метод одевания для уравнения Цицейки и находим преобразование Дарбу, связываюшее два решения ( $v$ и $\bar{v}$ ) уравнения Цицейки. Оно зависит от двух полей $\alpha$ и $\gamma$, удовлетворяюших уравнениям типа Риккати с коэффициентами, зависяшими от поля $v$ и его производных. Показано, что уравнения одевания наиболее просты в терминах полей, определяющих проекционную матрицу [10]. Кроме того, мы показываем, что преобразование Мутара для спектральной задачи второго порядка, связанной с уравнениями Цицейки, следует из композиции преобразований Дарбу в методе одевания. В конце раздела получены преобразования Беклунда для уравнения Цицейки в удобной форме. Процедура получения модифицированного уравнения Цицейки описана в разделе 3 . Исключая поле $v$ и вспомогательное поле $\gamma$ из уравнения одевания, мы получаем модифицированное уравнение Цицейки для полей $u$ и находим преобразование Миуры, связьвающее поля $u$ и $v$. В конце раздела обсуждаются преобразования Беклунда для модифицированного уравнения.

\section{2. ОДЕВАНИЕ И ПРЕОБРАЗОВАНИЯ БЕКЛУНДА ДЛЯ УРАВНЕНИЯ ЦИЦЕЙКИ}

В этом разделе мы применим метод последовательного одевания для уравнений Цицейки, который позволит по известному частному решению этого уравнения получить новый класс точных решений. Для удобства запишем уравнение Цицейки в переменных $v=\exp (-\theta)$ :

$$
v_{x, t}=v^{2}+\frac{v_{t} v_{x}}{v}-\frac{\tau}{v}
$$

С уравнением (2) ассоциированы две $(3 \times 3)$-матричные спектральные задачи [11] для трехмерного вектора $\Psi$

$$
\left\{\begin{array}{l}
\Psi_{x}=L \Psi, \\
\Psi_{t}=A \Psi,
\end{array}\right.
$$


где

$$
\begin{aligned}
L & =\left(\begin{array}{ccc}
-v_{x} v^{-1} & \zeta & 0 \\
0 & 0 & \zeta \\
\zeta & 0 & v_{x} v^{-1}
\end{array}\right) \\
A & =\left(\begin{array}{ccc}
0 & 0 & \tau v^{-2} \zeta^{-1} \\
v \zeta^{-1} & 0 & 0 \\
0 & v \zeta^{-1} & 0
\end{array}\right)
\end{aligned}
$$

( $\zeta$ - спектральный параметр), условие совместности которых $\Psi_{x t}=\Psi_{t x}$ эквивалентно уравнению (2). При известном частном решении $v$ определим новое решение $\bar{v}$ уравнения Цицейки и вспомогательное поле $\bar{\Psi}$, удовлетворяющее системе уравнений

$$
\left\{\begin{array}{l}
\bar{\Psi}_{x}=\bar{L} \bar{\Psi} \\
\bar{\Psi}_{t}=\bar{A} \bar{\Psi}
\end{array}\right.
$$

где матрищы $\bar{L}$ и $\bar{A}$ зависят от полей $\bar{\Psi}$. В случае, когда поля $\Psi$ и $\bar{\Psi}$ связаны линейным преобразованием $\bar{\Psi}=T \Psi$, матрица $T$ удовлетворяет линейным дифференциальным уравнениям

$$
\begin{aligned}
T_{x} & =\bar{L} T-T L, \\
T_{t} & =\bar{A} T-T A .
\end{aligned}
$$

Для оператора Шредингера, ассоциированного с уравнением Кортевега-де Фриза, такое преобразование называется преобразованием Дарбу. Аналогичные преобразования, включающие преобразования полей нелинейных интегрируемых уравнений и вспомогательных полей ассоциированных с этими уравнениями вспомогательных спектральных задач, найдены рядом исследователей для многих интегрируемых уравнений (см., например, [12]). Однако в общем случае нахождение таких преобразований для интегрируемых уравнений затруднено. В этой работе для нахождения матрицы $T$ мы используем метод одевания [10], который позволяет по заданному в явном виде частному решению интегрируемых уравнений получить новое решение ( "поместить" солитон на частное решение). Здесь мы не конкретизируем явный вид полей и с помощью матричной задачи Римана с нулями находим матрицу $T$. Согласно этому методу мы ишем решение для $T$ в виде мероморфной матричной функции и для простоты рассматриваем ее полиномиальное представление. Прямые вычисления для одевания простейшего решения $v=1$ уравнения (2) (при $\tau=1$ ) до солитонного решения

$$
\bar{v}=1-\frac{3}{1+\operatorname{ch}\left(3\left(\frac{x}{\beta}+t \beta\right)\right)}
$$

показывают, что матрища $T$ является полиномом второго порядка по спектральному параметру. В то же время легко непосредственно убедиться, что такой вид матрицы не 
удовлетворяет уравнению (8) для произвольных полей $v$ и $\bar{v}$. Поэтому мы рассмотрели разложение более высокого порядка в виде

$$
T=\zeta^{3} T_{3}+\zeta^{2} T_{2}+\zeta T_{1}+T_{0} .
$$

Определение зависяших от координат и времени матриц $T_{i}(i=0,1,2,3)$ значительно упрошается редукшией $L-A$-пары (3)-(5):

$$
\begin{aligned}
& \Omega L(x, t, \zeta) \Omega^{-1}=L(x, t, \eta \zeta), \\
& \Omega A(x, t, \zeta) \Omega^{-1}=A(x, t, \eta \zeta),
\end{aligned}
$$

где постоянная $\eta$ является кубическим корнем из единицы и отличные от нуля элементы матрицы $\Omega$ имеют вид $\Omega_{11}=\eta^{2}, \Omega_{22}=\eta, \Omega_{33}=1$. Подставляя затем разложение (9) в уравнение (8), находим матрицу $T$ :

$$
T=\left(\begin{array}{ccc}
\alpha-\zeta^{3} & \frac{\alpha \beta \zeta}{\zeta_{1}^{3}} & \frac{\alpha \beta^{2} \zeta^{2}}{2 \zeta_{1}^{6}} \\
\frac{-2 \zeta^{2} \zeta_{1}^{3}\left(\alpha+\zeta_{1}^{3}\right)}{\alpha \beta} & -\zeta^{3}+\zeta_{1}^{3} & \beta \zeta \\
\frac{-2 \zeta \zeta_{1}^{6}\left(\alpha+\zeta_{1}^{3}\right)^{2}}{\alpha^{2} \beta^{2}} & \frac{-2 \zeta^{2} \zeta_{1}^{3}\left(\alpha+\zeta_{1}^{3}\right)}{\alpha \beta} & -\zeta^{3}+\frac{\zeta_{1}^{6}}{\alpha}
\end{array}\right),
$$

где поля $\alpha$ и $\beta=\gamma / v$ с параметром $\zeta_{1}$ определяются системой уравнений

$$
\begin{aligned}
\alpha_{x}+\frac{\alpha^{2} \gamma^{2}}{2 v^{2} \zeta_{1}^{6}}+\frac{2 v \zeta_{1}^{3}\left(\alpha+\zeta_{1}^{3}\right)}{\gamma} & =0 \\
\frac{\gamma_{x}}{v}+\zeta_{1}^{3}-\frac{\zeta_{1}^{6}}{\alpha} & =0 \\
\alpha_{t}+\frac{\alpha \gamma}{\zeta_{1}^{3}}+\frac{2 \tau\left(\alpha+\zeta_{1}^{3}\right)^{2}}{\gamma^{2}} & =0 \\
-\frac{\gamma v_{t}}{v^{2}}+\frac{\gamma_{t}}{v}-\frac{\gamma^{2}}{2 v \zeta_{1}^{3}}-\frac{2 \tau \zeta_{1}^{3}\left(\alpha+\zeta_{1}^{3}\right)}{v \alpha \gamma} & =0
\end{aligned}
$$

При этом преобразование полей $v$ имеет простой вид:

$$
\bar{v}=\zeta_{1}^{3} \frac{v}{\alpha} .
$$

Уравнения (13)-(17) "одевают" любое решение уравнения Цицейки. При заданном поле $v$ решения уравнений (13)-(16) определяют явный вид полей $\alpha$ и $\gamma$. Тогда новое решение $\bar{v}$ уравнения Цищейки сразу получается из соотношения (17). Уравнения (13)-(16) имеют элегантный вид в терминах элементов проекционных матриц. Из явного вида определителя матришы $T$

$$
\operatorname{det} T(\zeta)=\left(\zeta_{1}^{3}-\zeta^{3}\right)\left(\zeta_{1}^{3}+\zeta^{3}\right)^{2}
$$

следует, что ранг матрицы $T\left(\zeta=-\zeta_{1}\right)$ равен единице и она является проекционной матрицей. Поэтому согласно методу одевания [10] ее элементы представимы в виде

$$
T_{i k}\left(\zeta=-\zeta_{1}\right)=m_{i} n_{k}
$$

Отсюда следует важная теорема.

5 Теоретическая и математическая физика, т. 131, № 1, 2002 г. 
Tеорема. Решение уравнения (8) при $\zeta=-\zeta_{1}$ представимо в виде (19), где $\vec{m}$ $u \vec{n}$ являются соответственно решениями уравнений

$$
\left\{\begin{array} { l } 
{ \vec { m } _ { x } = \overline { L } ( \zeta = - \zeta _ { 1 } ) \vec { m } , } \\
{ \vec { m } _ { t } = \overline { A } ( \zeta = - \zeta _ { 1 } ) \vec { m } , }
\end{array} \quad \left\{\begin{array}{l}
\vec{n}_{x}=-L^{\mathrm{T}}\left(\zeta=-\zeta_{1}\right) \vec{n} \\
\vec{n}_{t}=-A^{\mathrm{T}}\left(\zeta=-\zeta_{1}\right) \vec{n}
\end{array}\right.\right.
$$

где индекс Т обозначает транспонирование матрицы.

ДокАЗАТЕЛЬСТво. Перейдем к проективным компонентам $\mu_{i}=m_{i} / m_{s}(i=1,2,3)$ с фиксированным индексом $s$, тогда из уравнения (7) получаем

$$
\left(\frac{m_{i}}{m_{s}}\right)_{x}=\sum_{k=1}^{3}\left(\bar{L}_{i k}\left(\zeta=-\zeta_{1}\right) \frac{m_{k}}{m_{s}}-\frac{m_{i}}{m_{s}} \bar{L}_{s k}\left(\zeta=-\zeta_{1}\right) \frac{m_{k}}{m_{s}}\right)
$$

Отсюда сразу следует первое из уравнений системы $(20)$, где матрица $\bar{L}\left(\zeta=-\zeta_{1}\right)$ определяется с точностью до единичной матрицы. Остальные уравнения выводятся аналогичным образом. Отметим, что при $\zeta=\zeta_{1}$ матрица $T$ имеет ранг 2 , и уравнения типа (20) можно получить для полей, определяюших разложение матришы, составленной из миноров матрицы $T\left(\zeta=\zeta_{1}\right)$.

Преобразование Дарбу в виде уравнений (13)-(16) можно переписать в иной форме. Дифференцируя последовательно уравнения из системы (20), включающие $n_{2, x}$ и $n_{2, t}$, по переменным $x, t$ и исключая поля $n_{1}, n_{3}$, мы получаем замкнутую систему уравнений для $n_{2}(x, t)$ в следуюшем виде:

$$
\begin{aligned}
& n_{2, x x}=n_{2, x} \frac{v_{x}}{v}+\zeta_{1}^{3} \frac{n_{2, t}}{v}, \\
& n_{2, x t}=v n_{2}, \\
& n_{2, t t}=n_{2, t} \frac{v_{t}}{v}+\zeta_{1}^{3} \frac{n_{2, x}}{v},
\end{aligned}
$$

где постоянная $\zeta_{1}$ играет роль спектрального параметра. Из вида матрицы $T(12)$, определения (19) компонент вектора $\vec{n}$ (с точностью до постоянного множителя) и уравнения (17) сразу следуют выражения для $\alpha, \beta$

$$
\alpha=-\zeta_{1}^{3} \frac{n_{2}^{2}}{n_{2}^{2}-2 n_{1} n_{3}}, \quad \beta=-2 \zeta_{1}^{2} \frac{n_{3}}{n_{2}}
$$

и одевание потенциалов

$$
\bar{v}=-v+2 \frac{n_{2, t} n_{2, x}}{n_{2}^{2}}
$$

в терминах полей $\vec{n}$. Три условия совместности трех скалярных уравнений (22), впервые полученных еще Цицейкой [6], эквивалентны уравнению (2). Отметим также, что пара Лакса в виде (22) и преобразование потенциалов (24) были найдены недавно [5] при анализе сингулярных многообразий этого уравнения. 
Для второго уравнения системы (22) известны преобразования Мутара, применяемые в последнее время для различных спектральных задач второго порядка. Покажем, что такие преобразования, позволяюшие по двум различным (с фиксированным $v)$ решениям уравнения (22) получить решение с новым потенциалом, сразу следуют из композиции преобразований Дарбу в методе одевания. Рассмотрим последовательные преобразования $v \rightarrow \bar{v}$ с помощью одевающей матрищы $T(\zeta)$ с параметрами $\zeta_{1}$ и $\bar{v} \rightarrow \overline{\bar{v}}$ с помошью одеваюшей матрищы $\bar{T}(\zeta)$ с параметрами $\zeta_{2}$. Такие преобразования эквивалентны преобразованию $v \rightarrow \overline{\bar{v}}$ с помошью матрицы $\overline{\bar{T}}(\zeta)=\bar{T}(\zeta) T(\zeta)$. Элементы $\bar{T}(\zeta), \overline{\bar{T}}(\zeta)$ выражаются соответственно через поля $\bar{n}, \overline{\bar{n}}$, определенные соотношениями $\bar{T}_{i k}\left(\zeta=-\zeta_{2}\right)=\bar{m}_{i} \bar{n}_{k}, \overline{\bar{T}}_{i k}\left(\zeta=-\zeta_{2}\right)=\overline{\bar{m}}_{i} \overline{\bar{n}}_{k}$ и подчиняюшиеся, следовательно, уравнениям

$$
\bar{n}_{x}=-\bar{L}^{T}\left(\zeta=-\zeta_{2}\right) \bar{n}, \quad \overline{\bar{n}}_{x}=-L^{T}\left(\zeta=-\zeta_{2}\right) \overline{\bar{n}}
$$

и аналогичным уравнениям по переменной $t$. Поэтому поле $\overline{\bar{n}}_{2}$ удовлетворяет системе $(22)$ с заменой $\zeta_{1} \rightarrow \zeta_{2}$, а $\bar{n}_{2}-$ той же системе с заменой $v \rightarrow \bar{v}, \zeta_{1} \rightarrow \zeta_{2}$. Тогда поля $\overline{\bar{n}}$ определяются (с точностью до несущественного множителя) отношением компонент матрицы $\bar{T}(\zeta) T(\zeta)$, что и позволяет найти явное выражение полей $\bar{n}$ через поля $n, \overline{\bar{n}}$. Здесь мы приведем явный вид преобразования для полей $n_{2}$ (преобразование потенциалов определяется уравнением (17)):

$$
\bar{n}_{2}=\frac{a}{n_{2}}, \quad a=\frac{v n_{2}\left(\zeta_{1}^{3}-\zeta_{2}^{3}\right) \overline{\bar{n}}_{2}+2 n_{2, x} \zeta_{2}^{3} \overline{\bar{n}}_{2, t}-2 n_{2, t} \zeta_{1}^{3} \overline{\bar{n}}_{2, x}}{v\left(\zeta_{1}^{3}+\zeta_{2}^{3}\right)},
$$

где функция $а$ подчиняется простым уравнениям

$$
\begin{aligned}
a_{x} & =n_{2, x} \overline{\bar{n}}_{2}-n_{2} \overline{\bar{n}}_{2, x}, \\
a_{t} & =n_{2} \overline{\bar{n}}_{2, t}-n_{2, t} \overline{\bar{n}}_{2} .
\end{aligned}
$$

Обсудим преобразования Беклунда для уравнения (2). Согласно определению они представляются парой уравнений в частных производных для полей $v$ и $\bar{v}$ таких, что исключение $\bar{v}$ (или $v$ ) из этой пары дает уравнение Цицейки для полей $v$ (или $\bar{v}$ ). Попытки получения такой пары предпринимались рядом исследователей (см., например, [13]). Обзор литературы по этому вопросу и обсуждение полученных ранее результатов приведены в работе [5], авторы которой, по их мнению, нашли эффективные преобразования Беклунда. Такие преобразования могут быть сразу получены исключением полей $\alpha$ и $\gamma$ из соотношений (13)-(16). Мы получаем

$$
\begin{aligned}
& \bar{v}_{x}=\frac{\bar{v} v_{x}}{v}+\frac{\gamma^{2}}{2 v \zeta_{1}^{3}}+\frac{2 \bar{v}(v+\bar{v}) \zeta_{1}^{3}}{\gamma}, \\
& \bar{v}_{t}=\frac{\bar{v} v_{t}}{v}+2 \tau \zeta_{1}^{3} \frac{(v+\bar{v})^{2}}{v \gamma^{2}}+\frac{\bar{v} \gamma}{\zeta_{1}^{3}},
\end{aligned}
$$

где поле $\gamma=\zeta_{1}^{3} \int(\bar{v}-v) d x$. Прямым дифференцированием можно проверить, что условие совместности $\bar{v}_{x t}=\bar{v}_{t x}$ этой системы определяет функцию $\gamma_{t}$, дифференцирование по координатам которой с учетом определения $\gamma$ и уравнений $(29),(30)$ дает уравнение (2). 


\section{3. МОДИФИЦИРОВАННОЕ УРАВНЕНИЕ ЦИЦЕЙКИ}

Применим алгоритм размножения нелинейных уравнений, предложенный в [4], для получения нового интегрируемого уравнения, которое будет названо модифицированным уравнением Цицейки. Первым шагом этой схемы является исключение полей $v$ из уравнений одевания (13)-(16). В случае, когда рассматриваются нелинейные скалярные уравнения серии Кортевега-де Фриза и синус-Гордон, уравнение Буссинеска и $(2+1)$-мерные интегрируемые уравнения, исследованные в [14], преобразование Дарбу определяется одним вспомогательным полем, и процедура исключения потенциала из $L-A$-пары, восходящая к знаменитой работе Миуры [15], не представляет затруднений. Для уравнений типа Каупа-Буссинеска, эквивалентных системе двух нелинейных скалярных уравнений с $(2 \times 2)$-матричной $L-A$-парой, преобразование Дарбу зависит от двух вспомогательных полей, определенных как отношения компонент одеваюшей матрицы $T(\lambda)$ при двух различных значениях спектрального параметра $\lambda[16]$. В этом случае зависимость вспомогательных полей от $x$ и $t$ определяют четыре нелинейных уравнения первого порядка, два из которых позволяют найти выражения для полей уравнения Каупа-Буссинеска через вспомогательные поля (преобразование Миуры). Подстановка этих выражений в оставшиеся два уравнения приводит к интегрируемой системе двух нелинейных уравнений второго порядка с частными производными (модифицированному уравнению Каупа-Буссинеска) [16].

В рассматриваемом нами случае одевание решений уравнения Цицейки осушествляется двумя полями $\alpha$ и $\gamma$, и применение процедуры исключения поля $v$ из уравнений (13)-(16) для получения замкнутого уравнения только для одного поля осушествляется следуюшим образом. Вначале подставим выражение для поля $v$

$$
v=\frac{\alpha \gamma_{x}}{\zeta_{1}^{3}\left(\zeta_{1}^{3}-\alpha\right)}
$$

полученное из уравнения (14), в (13), (15), (16). Полученная система трех уравнений оказывается полной, поскольку после такой подстановки условие совместности системы (13), (15) эквивалентно уравнению (16). Поэтому для вывода нового интегрируемого уравнения мы используем только (13)-(15). Уравнение (15) является полиномом третьего порядка относительно поля $\gamma$ и определяет функцию $\gamma=\gamma\left(\alpha, \alpha_{t}\right)$ в явном виде. Подстановка ее и выражения (31) в (13) приводит к уравнению второго порядка для поля $\alpha$, которое и является модифицированным уравнением Цицейки. Эквивалентной записью этого уравнения является продифференцированное по $x$ уравнение $(15)$, где поля $\gamma$ и $\gamma_{x}$ после подстановки (31) в уравнения (15) и (13) можно выразить алгебраическим образом.

Упрошение записи модифицированного уравнения Цицейки достигается последовательными заменами

$$
\gamma_{x}=b \gamma\left(\alpha-\zeta_{1}^{3}\right), \quad \alpha=f(u)
$$


И

$$
\begin{array}{cl}
b=\frac{f_{u}\left(B+2 u_{x}\right)}{6 f\left(f+\zeta_{1}^{3}\right)}, & B=B\left(u_{x}\right), \\
\gamma=-\frac{f_{u}\left(A+2 u_{t}\right) \zeta_{1}^{3}}{3 f}, & A=A\left(u_{t}\right),
\end{array}
$$

где функция $f(u)$ удовлетворяет дифференциальному уравнению $f_{u}^{3}=-54 f^{2}\left(f+\zeta_{1}^{3}\right)^{2}$. Решение последнего

$$
f=-\frac{1}{2}\left(\frac{\wp_{u}}{2}+\zeta_{1}^{3}\right)
$$

выражается через функцию Вейерштрасса $\wp(u)$ :

$$
\wp_{u}^{2}=4\left(\wp^{3}+\zeta_{1}^{6}\right)
$$

В результате мы получаем модифицированное уравнение Цицейки для полей $u$ в следуюшем виде:

$$
u_{x t}=\frac{A\left(u_{t}\right) B\left(u_{x}\right)\left(6 \zeta_{1}^{3}+\wp^{\prime}(u)\right)}{4 \wp(u)},
$$

где функции $A\left(u_{t}\right)$ и $B\left(u_{x}\right)$ определяются алгебраическими уравнениями

$$
\begin{aligned}
1+\left(B\left(u_{x}\right)-u_{x}\right)\left(B\left(u_{x}\right)+2 u_{x}\right)^{2} & =0, \\
\tau+\zeta_{1}^{6}\left(A\left(u_{t}\right)-u_{t}\right)\left(A\left(u_{t}\right)+2 u_{t}\right)^{2} & =0 .
\end{aligned}
$$

Кроме того, из уравнения (31) мы находим преобразование Миуры

$$
v=\frac{1}{2} \wp(u)\left(A+2 u_{t}\right)\left(B+2 u_{x}\right)
$$

от уравнения (2) к уравнению (36), которое можно проверить прямыми вычислениями.

Преимушеством схемы размножения интегрируемых уравнений является возможность получения одеваюших цепочек, которые представляют собой преобразования Беклунда для модифицированного уравнения Цицейки с параметрами $\zeta$ и $\bar{\zeta}_{1}$. Для нахождения явного вида этих преобразований подставим преобразование Миуры (39) в преобразование Дарбу (17) и преобразование Беклунда (29) для уравнения Цицейки, считая $\alpha$ выраженным согласно (32). В результате мы получаем преобразование Беклунда для модифицированного уравнения в следуюшем виде:

$$
\begin{aligned}
\bar{u}_{t}= & \frac{-\bar{g}^{3} \bar{\zeta}_{1}^{9}+2 \bar{\zeta}_{1}^{6} \tau+\bar{\wp}^{3} \tau}{3 \bar{g}^{2} \bar{\zeta}_{1}^{6} \bar{\wp}^{2}}-\frac{\left(\bar{g}^{3} \bar{\zeta}_{1}^{3}+2 \tau\right) \bar{\wp}_{\bar{u}}}{6 \bar{\zeta}_{1}^{3} \bar{\wp}^{2} \bar{g}^{2}}, \\
\bar{u}_{x}= & -\frac{1}{12 \bar{g} \bar{\wp}^{2} \tau \wp^{2}}\left(A-u_{t}\right)\left(u_{x}-B\right)\left(2 \zeta_{1}^{3}+\wp_{u}\right)^{2} \times \\
& \times\left(\frac{\bar{g}^{3}\left(2 \bar{\zeta}_{1}^{3}+\bar{\wp}_{\bar{u}}\right)^{2}}{16}-\frac{8 \zeta_{1}^{9} \wp^{3}\left(2 \bar{\zeta}_{1}^{3}-\bar{\wp}_{\bar{u}}\right)\left(A+2 u_{t}\right)^{3}\left(B+2 u_{x}\right)^{3}}{\left(2 \zeta_{1}^{3}+\wp_{u}\right)^{3}}\right),
\end{aligned}
$$

где использовано обозначение

$$
\bar{g}=2 \zeta_{1}^{3} \int \frac{\wp\left(A+2 u_{t}\right)\left(B+2 u_{x}\right) 6\left(\bar{\zeta}_{1}^{3}+\bar{\wp}_{\bar{u}}\right)}{\left(2 \zeta_{1}^{3}+\wp_{u}\right)\left(2 \bar{\zeta}_{1}^{3}+\bar{\wp}_{\bar{u}}\right)} d x .
$$

Таким образом, мы показали эффективность метода одевания для построения преобразований Дарбу и применения процедуры размножения к спектральной задаче третьего порядка для одного скалярного уравнения. 
ЗАмЕчАнИЕ. Осенью 2000г., когда основные результаты работы были получены, В.В. Соколов любезно сообшил нам, что им также найдено модифицированное уравнение Цищейки и преобразование Миуры прямым методом, который не позволяет найти преобразование Беклунда. В присланном им варианте уравнение содержало функции, удовлетворяюшие определенному нелинейному уравнению.

Благодарности. Работа вьполнена при частичной поддержке РФФИ, грант №0001-00366, и гранта INTAS 99-1782.

\section{Список литературы}

[1] Э. Шредингер. Избранные труды по квантовой механике. М.: Наука, 1976.

[2] Х. Грин. Матричная квантовая механика. М.: Мир, 1968.

[3] А. П. Веселов, А. Б. Шабат. Функц. анализ и его прилож. 1993. Т. 27. № 2. С. 1.

[4] А. Б. Борисов, С. А. Зьъов. ТМФ. 1998. Т. 115. № 2. С. 199.

[5] R. Conte, M. Musette. J. Math. Phys. 1999. V. 40. P. 2092.

[6] G. Tzitzéica. C. R. Acad. Sci. Paris. 1907. V. 144. P. 1257.

[7] R. K. Dodd, R. K. Bullough. Proc. Roy. Soc. London. A. 1976. V. 351. P. 499.

[8] А. В. Жибер, А. Б. Шабат. ДАН СССР. 1979. Т. 247. С. 1103.

[9] А. В. Михайлов. Письма в ЖЭТФ. 1979. Т. 30. С. 443.

[10] В. Е. Захаров, А. Б. Шабат. Функц. анализ и егоприлож. 1979. Т. 13. С. 13; В. Е. Захаров, С. В. Манаков, С. П. Новиков, Л. П. Питаевский. Теория солитонов: Метод обратной задачи. М.: Наука, 1980.

[11] A. P. Fordy, J. Gibbons. J. Math. Phys. 1981. V. 21. P. 2508.

[12] V. B. Matveev, M. A. Salle. Darboux Transformation and Solitons. Berlin: Springer, 1991.

[13] C. С. Сафин, Р. А. ШІарипов. ТМФ. 1993. Т. 95. С. 146.

[14] А. В. Юров. ТМФ. 1999. Т. 119. № 3. С. 419.

[15] R. M. Miura. J. Math. Phys. 1968. V. 9. № 8. P. 1202.

[16] A. B. Borisov, M. V. Pavlov, S. A. Zykov. Physica D. 2001. V. 151-152. P. 104.

Поступила в редакцию 28.XII.2000 г. 